
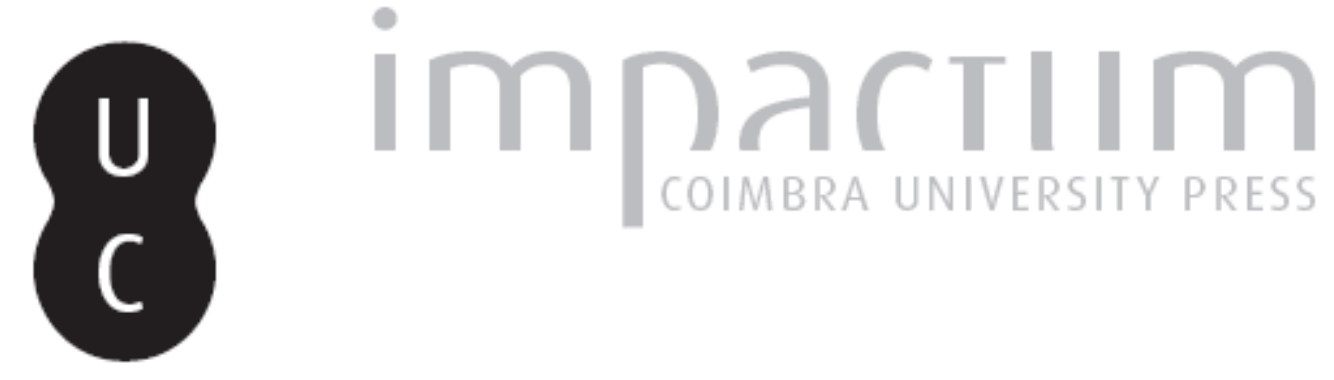

\title{
Consecuencias geomorfológicas del abandono agrícola en la cuenca del Guiniguada (Gran Canaria, Islas Canarias): aplicación a la conservación del patrimonio de bancales, una tesis doctoral de Lidia Romero
}
Autor(es):
Lourenço, Luciano
Publicado por:
Associação Portuguesa de Riscos, Prevenção e Segurança; Imprensa da Universidade de Coimbra
URL persistente:
URI:http://hdl.handle.net/10316.2/38082
DOI:
DOI:http://dx.doi.org/10.14195/1647-7723_22_24

Accessed : $\quad$ 26-Apr-2023 14:24:11

A navegação consulta e descarregamento dos títulos inseridos nas Bibliotecas Digitais UC Digitalis, UC Pombalina e UC Impactum, pressupõem a aceitação plena e sem reservas dos Termos e Condições de Uso destas Bibliotecas Digitais, disponíveis em https://digitalis.uc.pt/pt-pt/termos.

Conforme exposto nos referidos Termos e Condições de Uso, o descarregamento de títulos de acesso restrito requer uma licença válida de autorização devendo o utilizador aceder ao(s) documento(s) a partir de um endereço de IP da instituição detentora da supramencionada licença.

Ao utilizador é apenas permitido o descarregamento para uso pessoal, pelo que o emprego do(s) título(s) descarregado(s) para outro fim, designadamente comercial, carece de autorização do respetivo autor ou editor da obra.

Na medida em que todas as obras da UC Digitalis se encontram protegidas pelo Código do Direito de Autor e Direitos Conexos e demais legislação aplicável, toda a cópia, parcial ou total, deste documento, nos casos em que é legalmente admitida, deverá conter ou fazer-se acompanhar por este aviso.

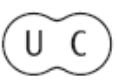




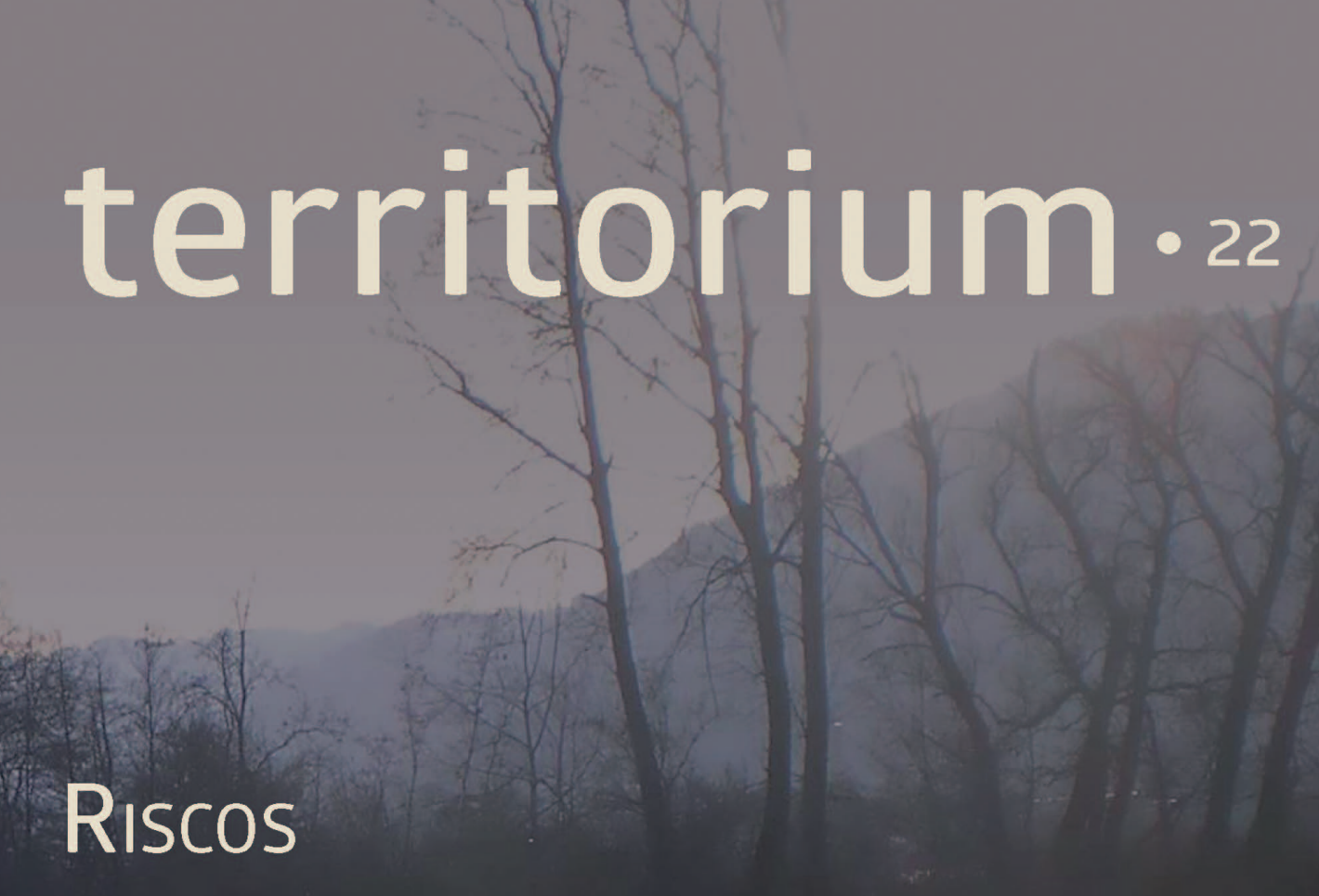

\section{TERRITÓRIOS DE CONVERGÊNCIA}

- Imprensa da Universidade de Coimbra Associação Portuguesa de:Riscos, Prevenção e Segurança 


\title{
RECENSÕES \\ CONSECUENCIAS GEOMORFOLÓGICAS DEL ABANDONO AGRÍCOLA EN LA CUENCA DEL GUINIGUADA (GRAN CANARIA, ISLAS CANARIAS): APLICACIÓN A LA CONSERVACIÓN DEL PATRIMONIO DE BANCALES, UNA TESIS DOCTORAL DE LIDIA ROMERO
}

\begin{abstract}
Luciano Lourenço
Departamento de Geografia e CEGOT Faculdade de Letras da Universidade de Coimbra
\end{abstract} luciano@uc.pt

La tesis doctoral de Lidia E. Romero Martín, con Dirección de la Dra. Emma Pérez-Chacón Espino, leída en Enero de 2015, se centra en el análisis de una problemática de carácter actual y global, donde son tratados distintos tipos de riesgos, por lo que es oportuna su divulgación. El abandono de la actividad agrícola es un fenómeno muy extendido en las áreas de montaña de todo el mundo, en las que se practicaba un tipo de agricultura de autosubsistencia y donde las vertientes fueron intensamente modificadas mediante el sistema de bancales. Con el abandono de la actividad agrícola se intensifica la actuación de un conjunto de procesos, en áreas de elevada vulnerabilidad, que determina una serie de riesgos para la conservación de los bancales, como es el caso de la cuenca del Guiniguada.

Esta tesis doctoral se ha desarrollado en el seno del Grupo de Investigación Geografía Física y Medio Ambiente, vinculado al Instituto de Oceanografía y Cambio Global (IOCAG) de la Universidad de Las Palmas de Gran Canaria. Se enmarca en una de las líneas de investigación del mencionado grupo que tiene como objetivo el análisis de las consecuencias ambientales de los cambios de usos del suelo, entre los que destaca, por su magnitud e incidencia ambiental y patrimonial, el abandono agrícola. Asimismo se enmarca en el proyecto de investigación "Estudio y proposición de actuaciones para la conservación y restauración de las terrazas agrícolas en el ámbito territorial del Proyecto Guiniguada". que forma parte del Proyecto Piloto Urbano Guiniguada financiado por la Unión Europea. Y se vincula con el proyecto europeo TERRISC "Recuperación de Paisajes de Terrazas y Prevención de Riesgos Naturales", perteneciente a la Iniciativa Comunitaria Interreg III-B Sudoeste Europeo.

Para hacer la lectura más amena, esta reseña está estructurada en cinco apartados. En el primero se presenta la temática y la estructura de la tesis; en el segundo, los objetivos planteados; en el tercero, la metodología empleada; en el cuarto, las conclusiones más relevantes $y$, finalmente, en el quinto, una valoración personal sobre la autora de esta reseña.

\section{Contexto y Estructura}

El abandono agrícola en el archipiélago canario alcanza su grado máximo a partir de la irrupción turística (década de los sesenta del siglo XX). Éste cambio de modelo económico está afectando a la conservación de los suelos, a la estabilidad de las vertientes, así como a la pérdida de calidad de los paisajes agrícolas tradicionales abancalados. Esos tres aspectos constituyen el objeto de análisis de este trabajo de investigación.

La tesis se estructura en ocho capítulos que, en esta reseña, aglutinamos en tres grandes bloques, en los que se recogen los siguientes aspectos:

1. En la primera parte se presenta la problemática objeto de estudio (abandono agrícola, erosión en vertientes agrícolas y conservación del patrimonio abancalado), se justifica la selección de dicha temática y se hace una amplia revisión conceptual y bibliográfica sobre los mismos. Con todo ello se aporta una visión general sobre la complejidad de los agrosistemas en vertiente, su fragilidad y la necesidad de su protección. También se realiza una introducción al área de estudio, se exponen los objetivos, las hipótesis y el diseño metodológico.

2. La segunda parte de esta investigación comprende el análisis de los resultados obtenidos. En el capítulo III se aborda la caracterización ecoantrópica de la zona de estudio (cuenca del Guiniguada) en la que se presta especial atención a los factores que organizan el paisaje agrícola y su aprovechamiento humano. El capítulo IV se dedica al análisis de cambios recientes en la ocupación del suelo y, en especial, a la evolución de la superficie agrícola en bancales. En el $\mathrm{V}$ se analizan los procesos de erosión y estados de conservación de las vertientes de cultivo, y se aporta una clasificación de estados geomorfológicos de los campos de cultivo. En el VI se cuantifican los procesos de erosión según su tipología geomorfológica, y se analizan los factores que repercuten en la activación de los mismos y en sus estados de conservación. 
Y en el VII y último de los capítulos dedicados a la exposición de resultados se analiza la valoración patrimonial de los bancales como paso previo a su conservación y gestión.

3. La tercera parte de esta investigación se centra en los aspectos de discusión y valoración general a partir de los resultados obtenidos del apartado anterior. La discusión comprende tanto la revisión de los objetivos planteados y los métodos empleados, como la reflexión sobre las hipótesis de partida. También incorpora otros aspectos, entre los que se incluyen la contribución de esta tesis al conocimiento del proceso de abandono agrícola en la cuenca del Guiniguada, de sus consecuencias geomorfológicas en la dinámica de las vertientes, especialmente a escala de parcela y del abundante y rico patrimonio de bancales presente en la misma. La investigación concluye con la presentación de una serie de interrogantes que pueden ser el objeto de trabajos futuros y de mejoras relacionadas con la metodología empleada en el análisis de la dinámica erosiva de los campos de cultivo abandonados y con el método diseñado para la valoración y conservación de los espacios abancalados.

\section{Objetivos}

Esta tesis doctoral tiene como objetivo principal conocer las consecuencias geomorfológicas que derivan del abandono agrícola de las vertientes de la cuenca hidrográfica del Guiniguada, con el objeto de identificar aquellos indicadores de diagnóstico que faciliten la posterior gestión y conservación de su importante patrimonio de bancales. Además de este objetivo principal, se plantearon otros específicos, relacionados con el análisis de los cambios de coberturas y su repercusión en las pérdidas de suelo y con la caracterización del proceso de abandono de las vertientes cultivadas, especialmente de las ocupadas por bancales, todo ello a escala de cuenca; con la identificación y la caracterización de tipos de estados erosivos según la diversidad de situaciones ambientales y antrópicas existentes, a escala de campo de cultivo; y finalmente con el diseño y ensayo de un método que permita valorar la calidad para la conservación y establecer categorías de prioridad y viabilidad para la restauración de unidades de paisaje con presencia de bancales.

\section{Metodología}

Siendo el objeto de estudio un fenómeno complejo, en el que se entremezclan la descomposición de un sistema de explotación tradicional, la implantación de nuevos usos en el territorio y la dinámica natural de cada espacio, se aplica un procedimiento metodológico de igual categoría. Se combinan un enfoque pluriescalar (cuenca hidrográfica, vertiente, unidad de paisaje con presencia de bancales y campo de cultivo) y multitemporal (dinámica de ocupación del suelo desde 1960 hasta 2002).

La secuencia metodológica se estructura en tres apartados para los cuales se utilizan fuentes y técnicas concretas que a pasamos a enumerar.

1. Para el análisis del proceso de abandono de la superficie agrícola a escala de cuenca se utilizan, como fuente de información y herramienta de análisis, fotogramas aéreos y ortofotos de las fechas seleccionadas (1960, 1992 y 2002), la fotointerpretación y los Sistemas de Información Geográfica. Con la implementación de los SIG se calcula la evolución de las tasas de erosión según el modelo USLE, se realizan mapas de coberturas del suelo, de evolución de la superficie agrícola, de la superficie de bancales y de su abandono.

2. Para el estudio de las consecuencias geomorfológicas de dicho abandono sobre las vertientes $y$, posteriormente, a escala de campo de cultivo, se realizan sendas campañas de campo en las que se recoge la información contenida en sus respectivas fichas de inventario. En la primera de ellas se realiza un amplio muestreo que abarca la totalidad de la cuenca, y con él se recaba la información necesaria para la clasificación de los campos según estados erosivos y, también, se obtienen los criterios necesarios que permiten una selección más reducida sobre la que efectuar análisis de detalle posteriores. Y, en la segunda, se recoge información detallada de un grupo de campos representativos del catálogo de situaciones geomorfológicas y de estados de conservación que existen en el área de estudio. La información obtenida en el campo se vuelca en hojas de cálculo para el tratamiento estadístico (descriptivo, bivariado y multivariado) de las variables consideradas, así como la representación gráfica de los análisis estadísticos más concluyentes.

3. Y, para la realización de diagnósticos de estados de conservación, de calidad para la conservación y de fragilidad erosiva y propuestas de prioridad y viabilidad para la restauración de bancales, se selecciona una zona piloto en la que se identifican las unidades de paisaje con presencia de bancales (fotointerpretación y trabajo de campo), se diseña y cumplimenta una ficha de inventario para cada una de las unidades resultantes, se vuelca la información en su correspondiente hoja de cálculo, sobre la que se realizan los diagnósticos y valoraciones señaladas, con el empleo de sus correspondientes 
algoritmos. Finalmente, la digitalización de las unidades de bancales y la implementación del proceso en un SIG permiten el tratamiento de los datos, además de la salida gráfica y cartográfica de los resultados obtenidos.

\section{Conclusiones y Propuestas}

La investigación realizada ha permitido demostrar las dos hipótesis de partida. En primer lugar, que los cambios de coberturas del suelo experimentados en la zona de estudio en los últimos cincuenta años, han supuesto importantes cambios en el tradicional aprovechamiento agrícola de las vertientes y que el abandono de esa actividad redunda en el incremento de la vulnerabilidad erosiva de las vertientes. Y, en relación con la anterior, que esos cambios suponen una pérdida del patrimonio agrícola en bancales y un bloqueo de su multifuncionalidad, por lo que resulta de sumo interés establecer un método de valoración de dicho patrimonio que favorezca su conservación.

A continuación se muestran las principales conclusiones alcanzadas en esta investigación según los bloques temáticos abordados en la misma.

En lo que respecta al análisis de cambios de coberturas del suelo y los procesos inductores de cambios, se constata que, entre 1960 y 2002, el abandono agrícola ha resultado ser el proceso más importante entre los cambios de cobertura del suelo. También se ha podido demostrar que el proceso de abandono agrícola afecta intensamente a la superficie de bancales de esta cuenca, concretamente al $60 \%$ de la misma. Se ha perdido el $28 \%$ de ese paisaje cultural por procesos de "naturalización" (repoblación y/o recolonización natural) y “solarización”, mientras que el $18 \%$ restante se corresponde con la superficie abancalada en abandono en 2002. Se aprecia que el abandono agrícola se inicia en los geoambientes extremos de la cuenca (cumbre y costa) y se extiende posteriormente hacia las medianías. $Y$ se diferencian dos etapas en el proceso de abandono: entre 1960-1992, donde la reducción de la superficie agrícola es el cambio más significativo; y entre 1992-2002, donde el proceso urbanizador de las superficies agrícola, en unos sectores y el de naturalización, en otros, son los más importantes.

En relación con las consecuencias geomorfológicas del abandono agrícola sobre las vertientes, se demuestra la diversidad de condiciones ecológicas, modelos de campos, y grados de explotación en la que se encuentran las mismas, de lo que resulta la gran variedad de estados erosivos existente. Esa variedad se ha simplificado en cuatro categorías, denominadas en este trabajo como «tipos geomorfológicos», tipología que responde al estado de conservación de los campos de cultivo. También se ha podido determinar que los factores de vulnerabilidad erosiva varían de unos tipos a otros, según los modelos de campos, la tipología de procesos y los grados de explotación.

En el bloque de valoración del patrimonio de bancales se concluye con los siguientes aspectos: El patrimonio de bancales no está suficientemente valorado en Canarias, ni por las instituciones ni por la sociedad. Se desconoce su extensión, variedad y estado de conservación. Se ha diseñado y verificado una metodología que permite su puesta en valor, así como la selección de unidades de bancales merecedoras de conservación y de restauración preferente. Dicha metodología resulta ser una herramienta útil para la planificación y gestión de los paisajes de bancales.

Con todo lo expuesto anteriormente se constata que la desagrarización y la consecuente contracción de la superficie agrícola tienen su fiel reflejo espacial en el predominio de los paisajes agrícolas del abandono, y en la pérdida de un paisaje agrícola tradicional de alto valor patrimonial y ambiental como es el paisaje de bancales. La consecuencia inmediata de su abandono es el bloqueo de la multifuncionalidad de estos agrosistemas, con la desorganización del sistema de vertientes, y la pérdida irreversible de recursos naturales (suelo, agua, vegetación natural y cultivada) y culturales (bienes patrimoniales tangibles e intangibles y de identidad cultural de un pueblo). Además del bloqueo de sus funciones de prevención y/o mitigación ante los riesgos naturales y mixtos (erosión, avenidas fluviales, movimientos en masa, incendios forestales y cambio climático).

Entre las perspectivas que se abren de trabajo a realizar en el futuro se plantea la conveniencia de seguir profundizando en el análisis de la dinámica erosiva de los bancales con la incorporación de variables ambientales y antrópicas que no se han tenido en cuenta en esta tesis. Entre las primeras cabe citar algunas relacionadas con su hidrología (capacidad de infiltración), la erodabilidad del suelo (estabilidad de los agregados), la recolonización vegetal y la erosividad de las lluvias (relación entre la frecuencia y magnitud de los procesos de erosión y el número de episodios lluviosos de alta energía). Y entre las segundas: categorías de edad de abandono, prácticas agrícolas y de conservación de suelos y uso posterior al abandono. Y, para poder dimensionar, desde el punto de vista espacial, los estados erosivos detectados se señala la conveniencia de incorporar las variables de vulnerabilidad erosiva de los tipos geomorfológicos en un modelo geomorfológico a escala de cuenca.

En lo que respecta al método propuesto para la valoración y conservación de los espacios abancalados se apuntan una serie de mejoras encaminadas a que la clasificación, los diagnósticos y las propuestas de restauración y gestión sean lo más completas posible, habida cuenta de la multifuncionalidad de estos paisajes culturales y patrimoniales. 
El trabajo debe continuar con la aplicación de dicha metodología valorativa y propositiva a toda la cuenca del Guiniguada, pues el ensayo se realiza en un sector de la misma, para la validación final de dicho procedimiento, habida cuenta de que las características de su patrimonio abancalado se diversifican en el resto de sectores de la cuenca y a efectos de su aplicabilidad a otras áreas abancaladas del archipiélago canario y de territorios afines, como el resto de la Macaronesia. Dicha ampliación también se justifica por la necesidad de obtener un diagnóstico de estados de conservación del patrimonio de bancales de un área geográfica con entidad propia como es la cuenca del Guiniguada.

Entre las reflexiones finales que presenta la autora de esta investigación se destacan: la necesidad de contar con un inventario del patrimonio de bancales de Canarias que favorezca su conocimiento, su difusión, su valoración y conservación entre los distintos sectores sociales (instituciones relacionadas con el territorio, el patrimonio, el desarrollo rural, la actividad agrícola, la educación, el turismo, la investigación, los habitantes de las islas y los turistas).

En un territorio como el archipiélago canario, superpoblado, con alta dependencia alimentaria del exterior y con un motor económico que es y parece que seguirá siendo el turístico, la actividad turística debe ir encaminada a la mejora de la calidad de las islas como destino turístico, en un nuevo modelo de explotación territorial en el que se integren el resto de los sectores productivos y la totalidad del territorio insular como componentes de la oferta turística.

Desde este trabajo se aboga por una protección activa de los agrosistemas abancalados en los que se debe mantener, en unos casos, y recuperar, en otros, aquella actividad productiva que contemple el valor de mantener un paisaje, recuperando e integrando viejos y nuevos sistemas de lucha contra la erosión y de sistemas de recarga de los acuíferos como son los paisajes agrícolas en terrazas. Por ello se considera que recuperar este singular legado cultural de gestión de espacios agrarios y adaptarlos al nuevo modelo económico y de intervención territorial en el marco de la sostenibilidad debe ser una de las prioridades de sus políticos y de sus habitantes. A su vez, es competencia de los científicos y de los docentes analizar y difundir los múltiples beneficios que implican la conservación y recuperación de los agrosistemas en bancales, con el objeto de recuperar la calidad de dichas islas como destino turístico.

\section{Valoración Personal de la Autora de esta Tesis}

La temática abordada en esta tesis doctoral, aunque es tratada a escala local, presenta un gran interés a escala global. El abandono de las áreas dedicadas a la agricultura tradicional y el actual estado de degradación de los paisajes culturales de bancales es un problema socioambiental que afecta en la actualidad a muchas regiones montañosas del mundo.

Así, la primera impresión que esta tesis nos deja es la de una excelente contribución para el conocimiento de la cuenca del Guiniguada y para la conservación del patrimonio de bancales en Canarias, pues está sostenida por una impresionante cantidad de trabajo, que también resulta de un profundo y minucioso conocimiento del terreno, dos aspectos que cabe valorar.

Con respecto al procedimiento metodológico realizado, se destaca el volumen y diversidad de variables recogidas en las tres fichas de inventario diseñadas para los distintos bloques temáticos abordados, especialmente para la identificación, clasificación y caracterización de los campos según estados erosivos y para la clasificación, valoración y diagnóstico de las unidades de paisaje con presencia de bancales.

La cantidad de información recogida en esas fichas supone un número impresionante de horas de trabajo empleadas en el campo para su acopio y en el laboratorio para su posterior análisis y tratamiento estadístico y cartográfico. Los resultados de este trabajo se muestran a través de un importante muestrario de tablas, gráficos y mapas que acompañan el extenso trabajo de redacción elaborado por su autora, en el que, por otro lado, el nivel de detalle y minuciosidad hacen, en ocasiones, que la lectura del mismo sea un trabajo denso.

Otro aspecto de este trabajo que merece una valoración positiva es la correcta integración de fuentes, técnicas y métodos de trabajo usados que van desde los análisis geomorfológico, sedimentológico y estadístico, hasta la implementación de los SIG en diversos aspectos que se tratan en el mismo (dinámica de coberturas del suelo, caracterización ecoantrópica de la zona de estudio, valoración del patrimonio de bancales).

Por otra parte, el trabajo está apoyado en una base científica sólida y sostenido en una extensa bibliografía, no solo general, pero también local, de Canarias y de la cuenca del Guiniguada, además de profusamente ilustrado, por lo que no quedan dudas sobre la calidad de este estudio.

En esta corta síntesis de un impresionante trabajo sobre la valoración del patrimonio de bancales de la cuenca hidrográfica del Guiniguada, mucho quedó por decir, pero creo que es suficiente para estimular su lectura por parte de todos los que se interesan por la geomorfología, el abandono agrícola, la conservación de los suelos agrícolas o por el patrimonio de bancales, para mencionar apenas algunos de los aspectos abordados, por lo que no podemos dejar de felicitar Lidia Romero por esta brillante tesis y que muestra, también, el amor y la dedicación de Lidia por sus islas Canarias, así como su gusto por la geomorfología, que casi es una pasión cuando trata y habla de "sus" bancales. 